\title{
Características de morbilidad en funcionarios de una universidad estatal de Costa Rica, América Central
}

\author{
Lourdes Arce Espinoza \\ Investigadora independiente; arcelourdes1@gmail.com
}

Recibido 7-VI-2013 • Corregido 17-IX-2013 Aceptado • 17-IX-2013

\begin{abstract}
Morbidity is an important indicator of the causes of mortality in a population, and its study allows focusing preventive health activities. This report presents data on the medical service of a state university in Costa Rica whose participants were the employees who became outpatients from January 19, 2009 to December 14, 2012. I analized 2010 electronic medical files. The main findings were that $53 \%$ of the population had between 1-10 annual consultations, young adults and adults consult in the same proportion. There were weekly and monthly cycles, just like in other health services in Costa Rica. The occupation and socioeconomic status (presented in some other studies as determinants of morbidity) in this population are not different from those in the national population. Many consultations are related to poor hygiene, inadequate diet and a sedentary lifestyle as well as to poor postural hygiene.
\end{abstract}

KEY WORDS: Morbidity, outpatient, state university of Costa Rica, national health pattern.

\section{RESUMEN}

La morbilidad constituye un indicador importante de las causas de mortalidad de una población y permite enfocar las acciones en salud preventiva para disminuir los riesgos. Aquí presento datos del servicio médico de una universidad estatal de Costa Rica. Sus participantes fueron los funcionarios que acudieron a consulta del 19 de enero del 2009 al 14 de diciembre del 2012. Se analizó la población total consultante, usando como instrumentos 2010 expedientes médicos electrónicos, se realizó un reporte y posterior el análisis estadístico en el programa SPSS. Los hallazgos principales fueron que el 53\% de la población consultó entre 1 a 10 consultas anuales, los dos grandes grupos de edad: adultos jóvenes y adultos, consultan en igual proporción; hay ciclos semanales y mensuales, correspondientes a los reportados en otros servicios de salud a nivel nacional. Las causas de consulta coinciden en las reportadas a nivel nacional, por lo que se mantiene la hipótesis de que la ocupación y el nivel socioeconómico (presentados en algunos otros estudios como factores determinantes de la morbilidad) en esta población no presentan un patrón distinto con respecto al patrón nacional. Muchas de las consultas realizadas están relacionadas con pobres hábitos de higiene, mala dieta y sedentarismo, así como poca higiene postural.

PALABRAS CLAVE: Morbilidad, consulta externa , universidad estatal de Costa Rica, patrón nacional de salud.
El registro de las causas de consulta externa, conocido como morbilidad, comprende el proceso de recolección y evaluación de las enfermedades dadas en la población en un área determinada; es de vital importancia porque permite generar proyecciones de posibles causas de mortalidad y el desarrollo de programas de medicina preventiva (Moya, 2003; Vaca, 2003).

Existen algunas diferencias de las causas de morbilidad en la población, principalmente están relacionadas con el nivel socioeconómico, que está ligado con ciertas patologías, determinadas tanto por el acceso a los servicios de salud, hábitos y estilos de vida y ocupación laboral (Ortiz-Barboza, 2010). Un aspecto importante de recalcar es la inversión de la pirámide etaria, que actualmente presenta niveles más altos de población en los grupos de mayor edad, lo cual genera un perfil sociodemográfico y de morbi-mortalidad con alto riesgo e incidencia de enfermedades crónicas no transmisibles (Ministerio de Salud, 2010).

No existen estudios que permitan comparar la morbilidad entre funcionarios de las universidades estatales; solo se evidencia una investigación inicial de Arce 
y Monge (2008) realizado con funcionarios de la UNED, donde se presentan los datos de morbilidad, así como los ciclos según época del año y día de la semana. Esta institución cuenta con 2547 funcionarios en plazo fijo o propiedad y 457 funcionarios en servicios profesionales; para un total de 3004 funcionarios en total; con edades entre 18 y 78 años, son residentes principalmente del Gran Área Metropolitana, con nivel socioeconómico y preparación académica variable, que incluye el personal de apoyo y académicos. La gran mayoría son casadas y con antigüedad laboral en la institución entre 1 a 30 años.

En este se manejan dos hipótesis: la primera es que existen los ciclos semanales y mensuales, al igual que lo reportado por otros servicios de salud a nivel nacional y según reportan en otros países (Moya, 2003), la segunda es que factores como la ocupación laboral y nivel socioeconómico de los funcionarios universitarios, no muestra diferencia de la población nacional en cuanto a las causas de morbilidad.

\section{METODOLOGÍA}

La recolección de los datos se obtuvo del expediente médico electrónico de cada funcionario (fuente primaria de información), Los datos fueron tabulados en la hoja electrónica Excel y mediante el Programa Estadístico SPSS, versión 15,0 se realizaron los análisis estadísticos determinados.

Sujetos: Los sujetos de estudio fueron todos los 2010 expedientes médicos electrónicos de los pacientes de la consulta externa del servicio médico de la universidad estatal que consultaron entre el 19 de enero del 2009 y el 14 de diciembre del 2012, 1132 corresponden al sexo femenino y 878 al sexo masculino.

Procedimiento: La recolección de datos se llevó a cabo en dos etapas: la primera comprendió la elaboración del reporte que se obtuvo del expediente médico electrónico y la solicitud del número de funcionarios de cada departamento, pues este no se registra en el expediente electrónico, por lo cual este dato tuvo que ser solicitado a cada oficina, ya que en Recursos Humanos el dato se maneja por vicerrectoría y no por ambas; la segunda fue la codificación de las causas de consulta con base en el Código Internacional de Enfermedades (CIE) (Organización Mundial de la Salud, 2009).

A partir de este registro se procedió al análisis estadístico mediante la prueba de Chi-cuadrado, correlación y clusters. Para realizar el agrupamiento de las consultas se utilizó el procedimiento Análisis de conglomerados en dos fases. Variables incluidas en el análisis: Sexo, dependencia, grupo de edad y Grupo CIE10 (diagnóstico).

\section{RESULTADOS}

La cantidad de consultas por vicerrectoría y otros departamentos es mayor en las mujeres, excepto en la categoría de pensionados; el cual es mayor en los hombres (Fig. 1). La categoría de no disponible se refiere a los funcionarios que no tenían asignada dependencia u oficina en el expediente electrónico y cuya información no fue suministrada por la Oficina de Recursos Humanos.

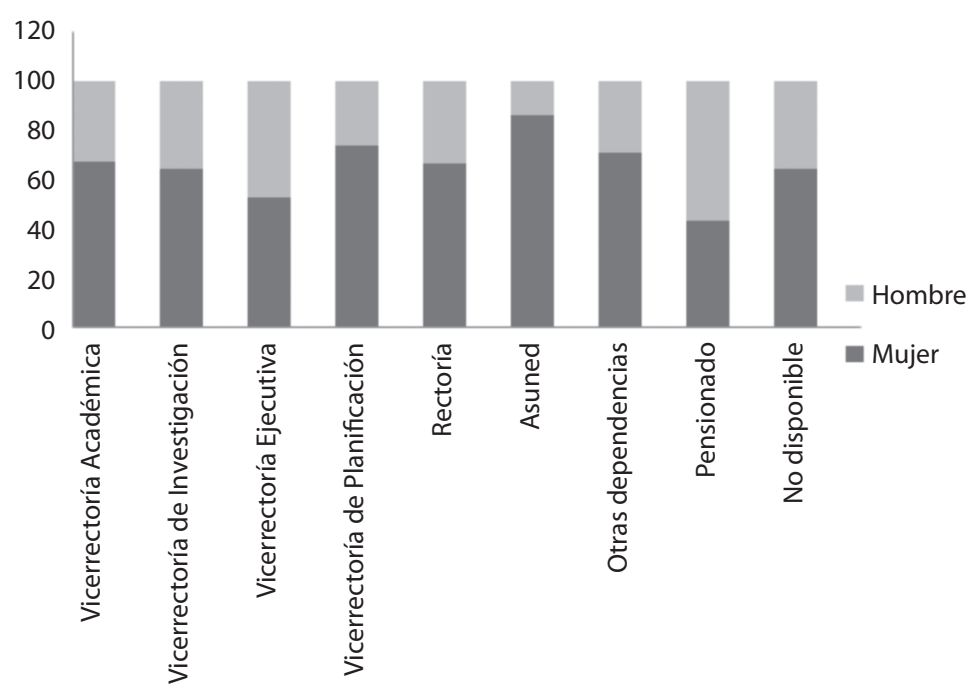

Fig. 1. Distribución de consultas por sexo según dependencia, 2009-2012 (cantidad acumulada de consultas en porcentajes). 
El grupo de edad que más consulta registra para cada una de las vicerrectorías u oficinas es el comprendido entre los 28 a 37 años. El grupo mayor de 58 años es el grupo de menos consultas realizadas (ver apéndice 1). La mayoría de los funcionarios tenía entre 18 y 57 años de edad al momento de la consulta, el promedio es de 39 años; aunque hubo extremos cercanos a los 78 años $\left(X^{2}=5\right.$ 950,242, Gl 4, p<0,001; Fig. 2).

El $53 \%$ de los pacientes realizó entre 1 y diez consultas durante el periodo de estudio. Aproximadamente el 33\% hizo entre 11 y 30 consultas, el resto una cantidad mayor, hasta un máximo de 128 consultas (Fig. 3).

Los días con mayor cantidad de consultas fueron lunes, martes y fue disminuyendo al acercarse el fin de semana $\left(X^{2}=513,868, \mathrm{Gl} 4, \mathrm{p}<0,001\right.$; Fig. 4).

La universidad cierra dos semanas en diciembre y enero; una semana en marzo o abril, por Semana Santa, por lo cual puede considerarse que la cantidad de consultas es relativamente estable a lo largo del año, con un aumento en julio y octubre debido a las campañas de salud, en julio por la campaña preventiva de ultrasonidos; en octubre campaña preventiva del cáncer de seno $\left(X^{2}=1\right.$ 749,605, Gl 11, p<0,001; Fig. 5).

Las cinco principales causas de consulta son enfermedades del sistema respiratorio, factores que influyen en el estado de salud y contacto con los servicios de salud (que corresponde a adulto sano, certificado médico, control anual); enfermedades osteomusculares, enfermedades endocrinas, nutricionales y metabólicas; enfermedades del aparato digestivo $\left(X^{2}=30206,970, G l 19, p<0,001\right.$; Fig. 6).

Las incapacidades generadas según la consulta médica, fueron un total de 3715 , siendo el $38 \%$, producto de las enfermedades del sistema respiratorio (Fig. 7).

El promedio de consultas por Vicerrectoría (forma global) no sobrepasa las 7 consultas establecidas como

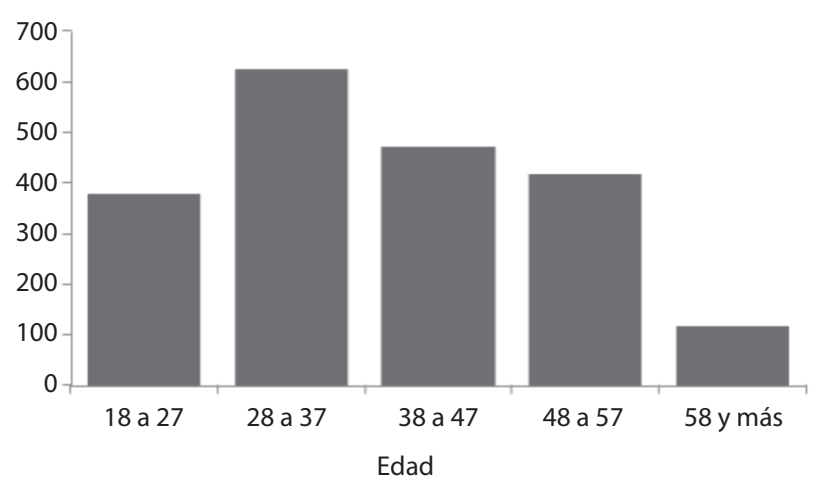

Fig. 2. Grupos de edad en la consulta externa, 2009-2012.

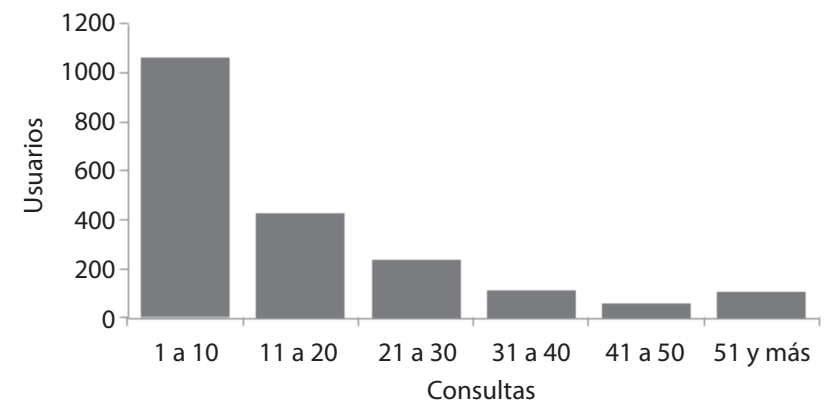

Fig. 3. Número de consultas por usuario en la consulta externa, 2009-2012.

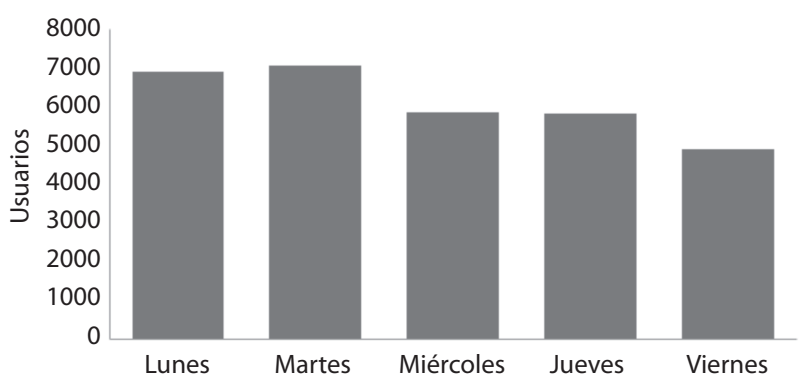

Fig. 4. Distribución de la consulta externa, según el día de la semana, 2009-2012 (cantidad acumulada de consultas).

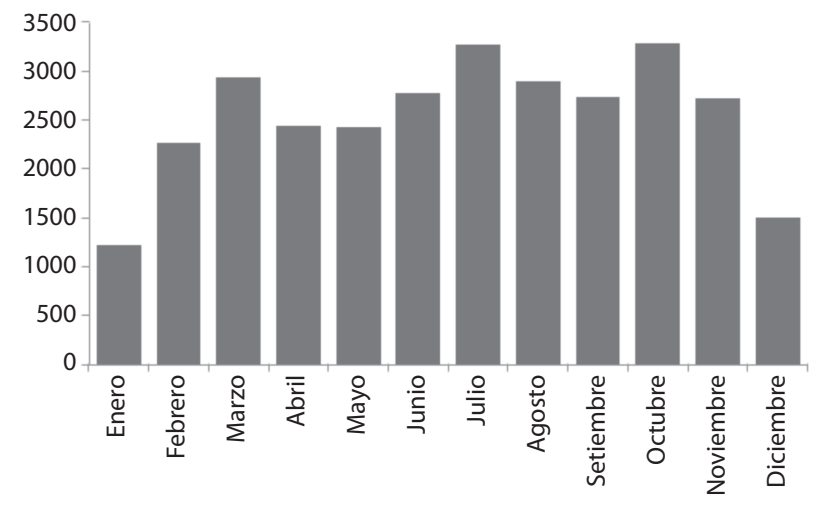

Fig. 5. Distribución mensual de la consulta externa, 2009-2012 (cantidad acumulada de consultas).

límite normal de atención en los centros de salud por usuario por año; sin embargo, en cada Vicerrectoría se puede observar que más de dos departamentos u oficinas sobrepasan esta cifra; en el caso de la Vicerrectoría académica estos departamentos son: Centro de Información y Documentación Bibliográfica, Dirección de Extensión y Centro de Proyección Municipal. En la Vicerrectoría Ejecutiva: Dirección Financiera, Federación de Estudiantes, Oficina de Contabilidad, Tesorería, Oficina de Registro, Oficina de Recursos Humanos, Oficina de 


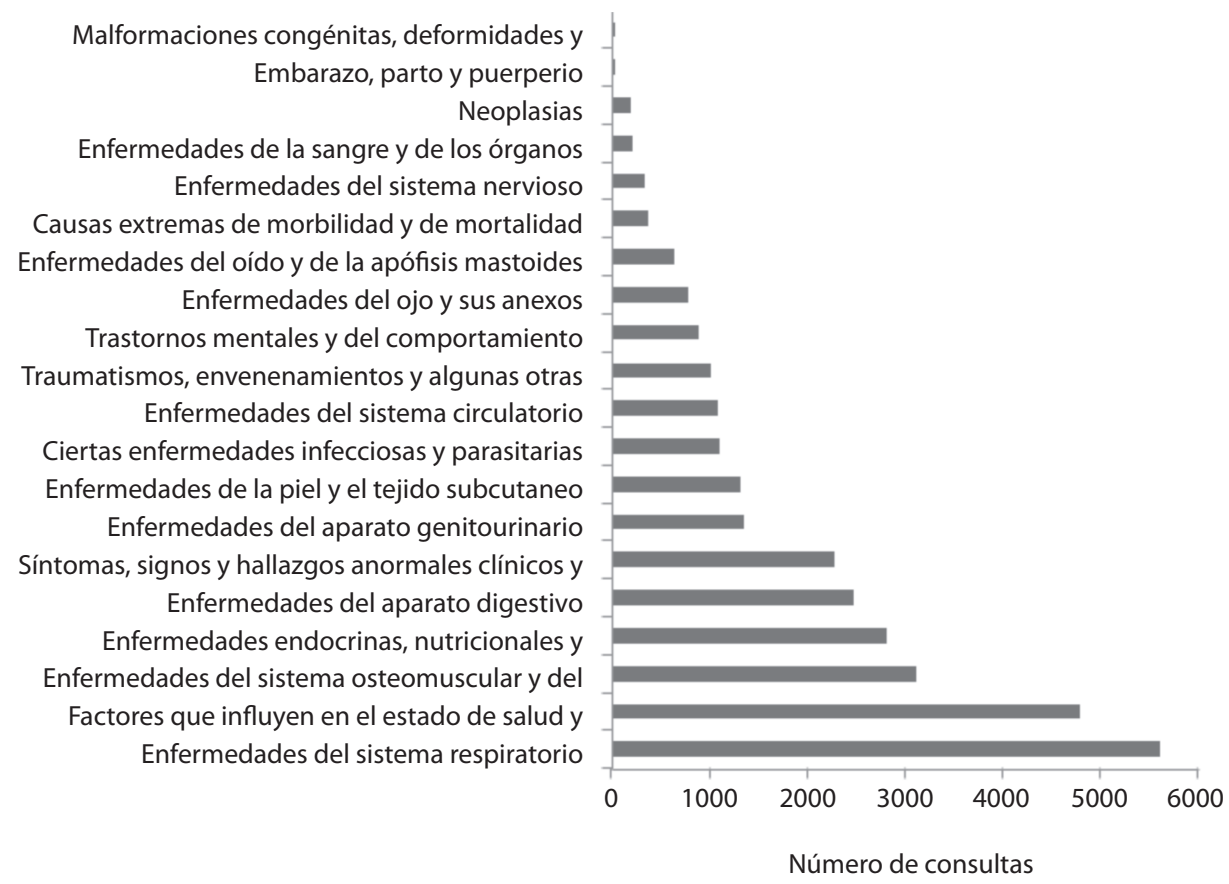

Fig. 6. Consultas por diagnósticos agrupados según capítulos del CIE.

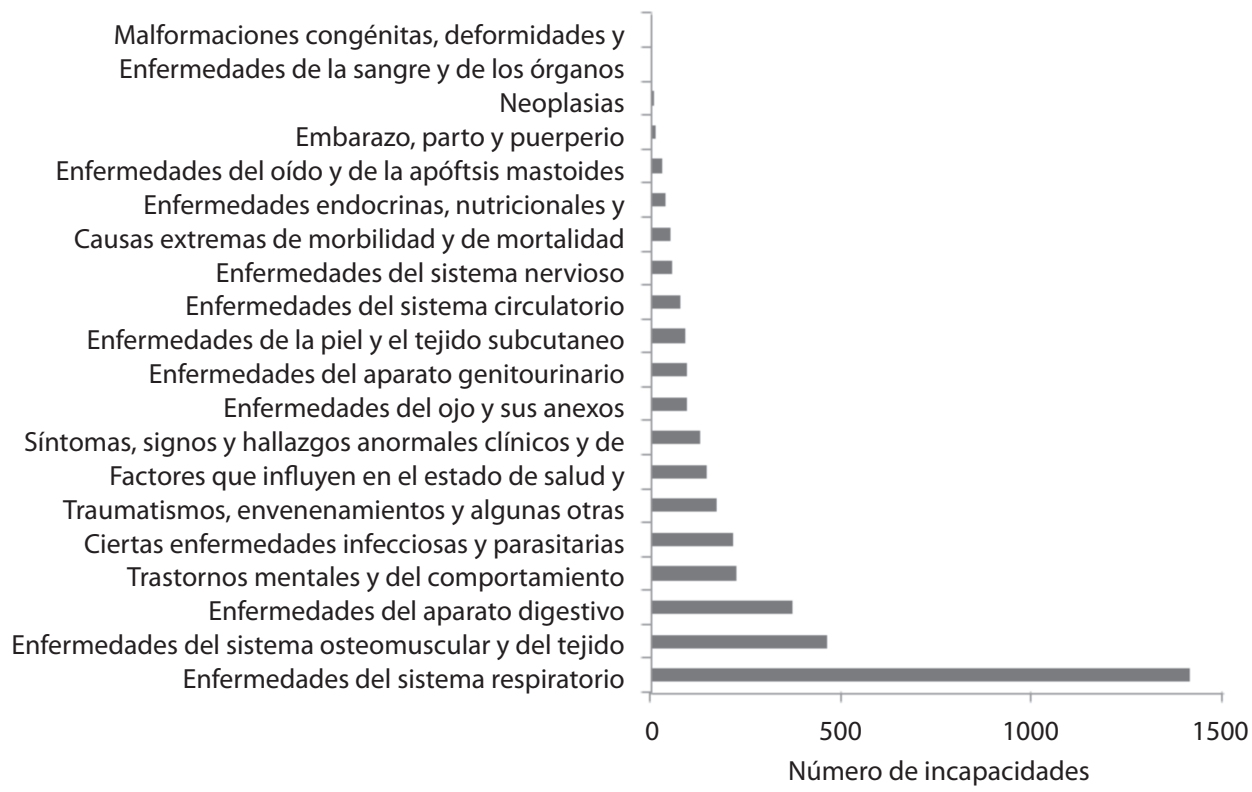

Fig. 7. Número de incapacidades otorgadas por diagnósticos agrupados según capítulos del CIE.

Contratación y Suministros y personal de la Vicerrectoría Ejecutiva. En la Vicerrectoría de Planificación: Centro de Planificación y Programación Institucional. En el caso de la Rectoría: Dirección de Tecnología y Comunicaciones y personal de Rectoría. En otras dependencias, encontramos el Consejo Universitario (Cuadro 1).
El grupo de edad de 28 a 37 años presenta según los primeros cinco diagnósticos más frecuentes reportados, el mayor número de consulta para cada uno de ellos. Los funcionarios mayores de 38 años reportan el $50 \%$ de las consultas por enfermedades endocrinas nutricionales y metabólicas. La mayoría de las consultas en 
CUADRO 1

Promedio de consultas anuales por departamento y por funcionario.

\begin{tabular}{|c|c|c|c|}
\hline Dependencia & Total consultas & Promedio consultas por año & $\begin{array}{l}\text { Promedio consultas anuales } \\
\text { por funcionario }\end{array}$ \\
\hline Vicerrectoría Académica: & 15221 & 3805 & 1,8 \\
\hline Accion Interu.Construcc. Oport & 72 & 18 & 2,3 \\
\hline Centro Capacitac. Educ. A Dist & 205 & 51 & 5,7 \\
\hline Centro de Educacion Ambiental & 98 & 25 & 3,5 \\
\hline Centro de Idiomas & 356 & 89 & 0,7 \\
\hline Centro de Operaciones Academ. & 398 & 100 & 6,2 \\
\hline Centro Inf., Doc. y Rec. Bibl. & 542 & 136 & 9,7 \\
\hline Desarrollo Gerencial & 13 & 3 & 0,5 \\
\hline Dir. Centros Universitarios & 1517 & 379 & 1,6 \\
\hline Direc. Prod. Materiales Didac. & 1192 & 298 & 6,3 \\
\hline Direccion de Posgrados & 601 & 150 & 4,1 \\
\hline Direccion Extension Universit. & 1500 & 375 & 8,0 \\
\hline Escuela Ciencias de La Admint. & 1061 & 265 & 1,2 \\
\hline Escuela Ciencias de La Educac. & 1640 & 410 & 1,0 \\
\hline Escuela Ciencias Exactas y Nat & 1532 & 383 & 1,0 \\
\hline Escuela Ciencias Soc. y Human. & 1281 & 320 & 0,8 \\
\hline Produccion Audiovisual & 494 & 124 & 4,3 \\
\hline Programa Coned & 277 & 69 & 5,8 \\
\hline Programa de Aprendizaje Linea & 60 & 15 & 1,5 \\
\hline Programa Gerontologia & 64 & 16 & 1,0 \\
\hline Programa Pace & 275 & 69 & 6,9 \\
\hline Programa Videoconferencia & 215 & 54 & 5,4 \\
\hline Proy. Formacion y Capac. Munic & 599 & 150 & 7,9 \\
\hline Vicerrectoria Academica & 1229 & 307 & 4,8 \\
\hline Vicerrectoría de Investigación: & 696 & 174 & 5,0 \\
\hline Prog. Invest. Fund. Educ. Dist & 109 & 27 & 5,5 \\
\hline Vicerrectoria de Investigacion & 587 & 147 & 4,9 \\
\hline Vicerrectoría Ejecutiva: & 10936 & 2734 & 7,6 \\
\hline Almacen General & 28 & 7 & 0,9 \\
\hline Centro de Salud Ocupacional & 48 & 12 & 4,0 \\
\hline Direccion Asuntos Estudiant. & 879 & 220 & 5,1 \\
\hline Direccion Editorial & 1755 & 439 & 7,0 \\
\hline Direccion Financiera & 126 & 32 & 7,9 \\
\hline Federación de Estudiantes Uned & 79 & 20 & 9,9 \\
\hline Oficina Control de Presupuesto & 412 & 103 & 5,2 \\
\hline Oficina de Contabilidad & 531 & 133 & 8,3 \\
\hline Oficina de Contratacion Y Sum. & 440 & 110 & 12,2 \\
\hline Oficina de Recursos Humanos & 1362 & 341 & 10,0 \\
\hline Oficina de Registro & 712 & 178 & 10,5 \\
\hline Oficina de Servicios Generales & 2652 & 663 & 7,5 \\
\hline Oficina de Tesoreria & 544 & 136 & 8,5 \\
\hline Oficina Distribucion y Ventas & 743 & 186 & 6,6 \\
\hline Vicerrectoria Ejecutiva & 625 & 156 & 9,8 \\
\hline Vicerrectoría De Planificación: & 647 & 162 & 4,4 \\
\hline Centro Inv. y Evaluacion Inst. & 230 & 58 & 3,4 \\
\hline
\end{tabular}


CUADRO 1 (Continuación)

\begin{tabular}{lccc}
\multicolumn{1}{c}{ Dependencia } & Total consultas & Promedio consultas por año & $\begin{array}{c}\text { Promedio consultas anuales } \\
\text { por funcionario }\end{array}$ \\
Centro Planif. y Progr. Inst. & 233 & 58 & 7,3 \\
Dir. Internac. y Cooperacion & 42 & 11 & 1,8 \\
Vicerrectoria De Planificacion & 142 & 36 & 5,9 \\
Rectoría: & 1710 & 428 & 7,2 \\
Agenda Joven & 5 & 1 & 0,2 \\
Dir. Tecnologia Inf. y Comun. & 1191 & 298 & 8,3 \\
Oficina Inst. Mercadeo y Com. & 167 & 42 & 5,2 \\
Rectoria & 347 & 87 & 9,6 \\
Otras Dependencias: & 785 & 196 & 5,5 \\
Aseuned & 129 & 32 & 3,6 \\
Auditoria & 207 & 52 & 5,2 \\
Consejo Universitario & 300 & 75 & 10,7 \\
Dir. Defensoria de Estudiantes & 56 & 14 & 3,5 \\
Oficina Juridica & 79 & 20 & 4,9 \\
Programa Simpli. Proc. Gob. Di & 14 & 4 & 1,8 \\
Totales & 29995 & & 2,8
\end{tabular}

cada patología son realizadas por las mujeres. Con una distribución de 60 a $65 \%$ en cada uno de ellos para las mujeres (Ver apéndices 2 y 3). La aplicación del procedimiento de los conglomerados con las variables de edad, sexo dependencia y diagnóstico CIE 10, da como resultado óptimo la clasificación de las consultas en cuatro grupos, distribuidos tal como se muestra en la figura 8 y apéndice 4. Las cuatro variables incluidas en el análisis son significativas, es decir, permiten diferenciar adecuadamente los casos que corresponden a cada grupo y que todas superan los valores críticos correspondientes.

\section{Grupo 1: Pacientes de edad promedio que laboran principalmente en dependencias de la Vicerrectoría Académica, con bastante igualdad en la distribución por género.}

Este es el grupo más pequeño, constituido por 5479 consultas ( $18 \%$ de los casos). Un $57 \%$ de los pacientes son mujeres. El 75\% están en el grupo de edad promedio: de 38 a 47 años. La mayoría (89\%) laboran en dependencias de la Vicerrectoría Académica. En orden de importancia, los diagnósticos más frecuentes de este grupo son:

- Enfermedades del sistema respiratorio (17\%)

- Factores que influyen en el estado de salud y contacto con los servicios de salud (14\%)

- Síntomas, signos y hallazgos anormales clínicos y de laboratorio, no clasificados en otra parte (13\%)
- Enfermedades del sistema osteomuscular y del tejido conectivo (10\%)

Grupo 2: Pacientes de edad promedio que laboran principalmente en dependencias de la Vicerrectoría Ejecutiva, con mayor proporción de mujeres.

El grupo 2 quedó conformado por 6596 consultas ( $22 \%$ del total). La mayoría de los pacientes labora en dependencias de la Vicerrectoría Ejecutiva (76\%) y el 74\% son mujeres. Un $90 \%$ de los pacientes tiene edades inferiores a los 48 años. Los tres diagnósticos más frecuentes en este grupo son:

- Enfermedades del sistema respiratorio (31\%)

- Enfermedades del sistema osteomuscular y del tejido conectivo (15\%)

- Factores que influyen en el estado de salud y contacto con los servicios de salud (12\%)

Grupo 3: Hombres de diferentes edades que laboran en dependencias de la Vicerrectoría Ejecutiva y Vicerrectoría Académica.

En este grupo clasifican 7654 consultas (25\% de los casos). Un $47 \%$ de los pacientes laboran en la Vicerrectoría Ejecutiva y un $39 \%$ en dependencias de la Vicerrectoría Académica. El $42 \%$ son jóvenes con edades entre 28 y 37 años, pero también hay un $41 \%$ que tienen entre 48 y 57 años. El $98 \%$ de los pacientes son hombres. 


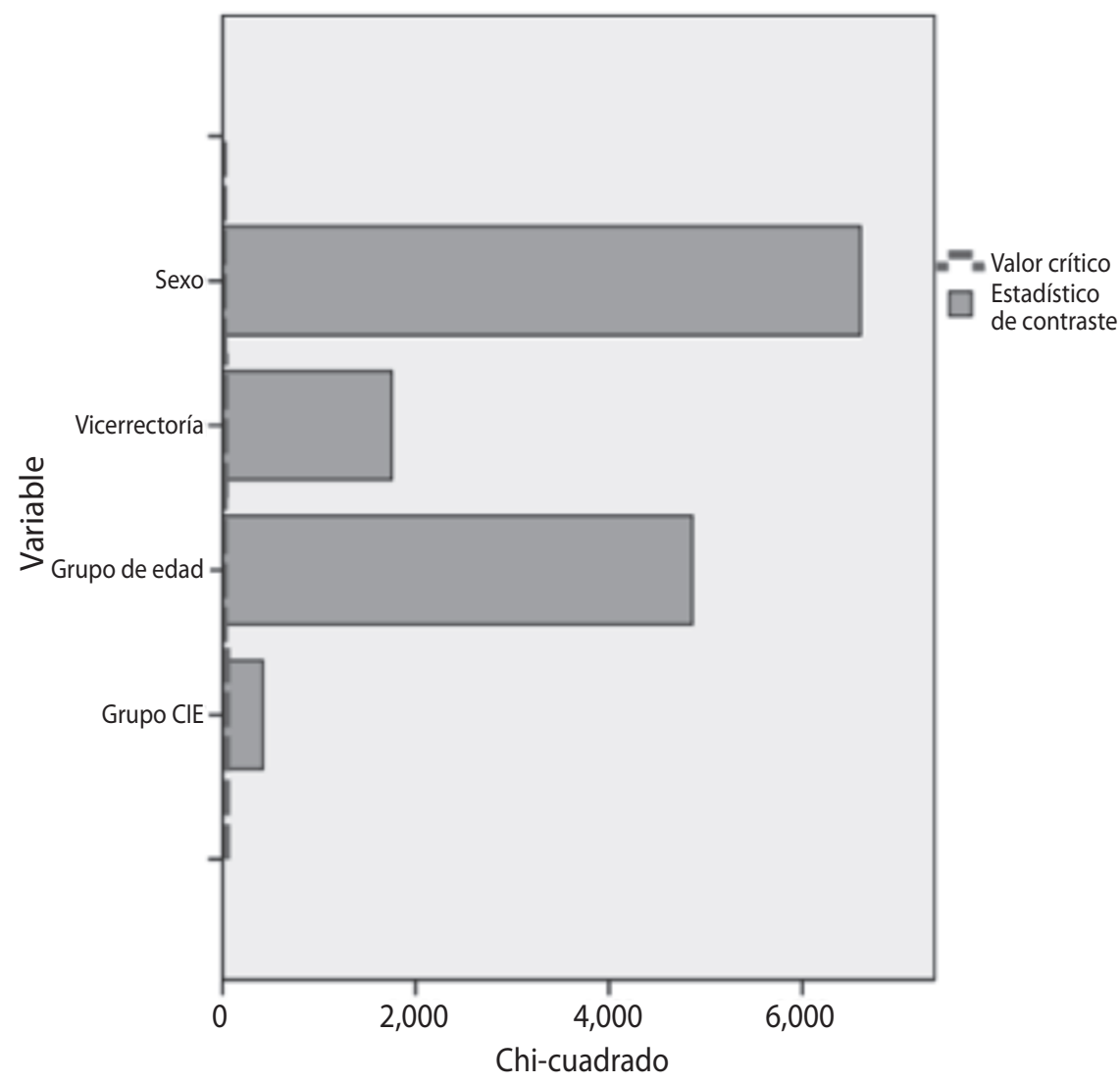

Fig. 8. Distribución de los conglomerados.

- Los diagnósticos más comunes en este grupo son los siguientes:

- Factores que influyen en el estado de salud y contacto con los servicios de salud (17\%)

- Enfermedades del sistema respiratorio (16\%)

- Enfermedades del aparato digestivo (10\%)

- Enfermedades del sistema osteomuscular y del tejido conectivo (9\%)

\section{Grupo 4: Mujeres de diferentes edades que laboran principalmente en dependencias de la Vicerrectoría Académica.}

Es el grupo más grande, conformado por 10745 consultas ( $35 \%$ del total). El $100 \%$ de las pacientes son mujeres y un $69 \%$ laboran en dependencias de la Vicerrectoría Académica. Un $47 \%$ tiene edades entre 28 y 37 años, y un $36 \%$ tiene entre 48 y 57 años. Sus diagnósticos más frecuentes son:

- Factores que influyen en el estado de salud y contacto con los servicios de salud (18\%)
- Enfermedades del sistema respiratorio (14\%)

- Enfermedades endocrinas, nutricionales y metabólicas (10\%)

- Síntomas, signos y hallazgos anormales clínicos y de laboratorio, no clasificados en otra parte (9\%).

\section{DISCUSIÓN}

Número de consultas por usuarios: el número de consultas por usuario se considera normal en la población, que puede presentar alguna patología o bien se realiza exámenes de rutina y chequeo anual, sin llegar a hacer policonsultante ("usuario que consulta más de siete veces al año, por iniciativa propia"), generalmente el galeno solicita exámenes de gabinete, según la exploración física, dejando una o varias citas de control para valoración de los resultados clínicos aunque puede también deberse a control de evolución al tratamiento terapéutico (Caja Costarricense de Seguro Social, 2002a). El sexo femenino reporta más consultas esto podría deberse a que las mujeres realizan más controles de su salud 
con mayor regularidad, mientras que los hombres se exponen a más riesgos y acuden en menos proporción al médico (Lorca, 2013).

Edad: los grupos de edad están distribuidos en adultos jóvenes que van de los 18 años a los 37 años, y adultos de 38 a más de 58 años; ambas poblaciones consultan en igual proporción; existiendo una correlación significativa entre la edad y la patología presentada. Se pudo observar que los adultos jóvenes, consultan más por chequeos médicos anuales, planificación familiar, trastornos digestivos, sobrepeso y obesidad, enfermedades de transmisión sexual y depresión (Organización Mundial de la Salud, 2011); mientras que los adultos consultan por causas de patologías en su mayoría crónicas, que podrían ser producto de hábitos y conductas que se desarrollaron durante la niñez, adolescencia y juventud y que se manifiestan en la edad adulta, tales como las enfermedades crónicas no transmisibles; que son resultado de dieta inadecuada, fumado, consumo de drogas, sedentarismo y pobres hábitos de higiene (Sepúlveda, 1994). La estrecha relación entre la edad y el desarrollo de ciertas patologías se debe a múltiples factores tales como: estilos de vida, procesos celulares, ambientales y genéticos, dando como resultado una disminución en la salud y aumento de la morbilidad (Alfieri \& Challem, 2007). Pudo observarse que el grupo de edad que más consulta es el comprendido entre los 27 a los 38 años, lo cual es consistente con el promedio de edad de los funcionarios, el cual es de 39 años.

Días de consulta: Los inicios de semana (lunes y martes) constituyen los días de más consulta médica por parte de los usuarios, igual que la tendencia nacional (Moya, 2003). Esto puede ser un efecto acumulativo del fin de semana, ya que muchos usuarios prefieren esperarse al lunes para consultar y no acudir a otro centro de salud (Arce \& Monge, 2008).

Distribución mensual de la consulta médica: durante todo el año se mantiene estable, excepto por dos descensos en el último mes (diciembre) y primer mes del año (enero) debido a las vacaciones institucionales, que corresponden a dos semanas de cada uno de ellos (Arce $\&$ Monge, 2008). Pueden observarse dos picos en julio y octubre lo cual se explicaría como producto de la campaña preventiva de ultrasonidos realizada en julio y campaña de prevención de cáncer de seno en octubre; lo cual dispara la demanda de consultas médicas para revisión de ambos estudios clínicos.
Morbilidad: Las enfermedades de las vías respiratorias de los funcionarios que laboran en oficinas ocupan el primer lugar de las consultas médicas, esto podría deberse al hacinamiento del personal en las oficinas, deficientes hábitos de higiene, poca o nula aplicación del protocolo estornudo y tos, además de una técnica pobre del lavado de manos (Arce \& Monge, 2011); las enfermedades del sistema osteomuscular, pueden explicarse por malas posturas, sedentarismo, por la ocupación que obliga al usuario a permanecer largas horas en una misma postura (Linero \& Rodríguez, 2012) y sobrepeso u obesidad (Esquivel \& Alvarado, 2009; Oliva, 2009); las patologías del sistema digestivo así como las nutricionales y metabólicas, están relacionadas con el sedentarismo, y la dieta (Oliva, 2009); las consultas sobre factores que influyen en el estado de salud y contacto con los servicios de salud, corresponden a exámenes anuales de control y certificados de ingreso, catalogándose esta consulta como "adulto sano".

Incapacidades según causa de consulta: el mayor número de incapacidades corresponde a las enfermedades de la vías respiratorias, lo cual es consistente con el Informe Nacional de la Caja Costarricense del Seguro Social (2002b), esto debido a que muchos de estos cuadros son virales, por lo cual existe mayor predisposición al contagio en espacios cerrados (Organización Mundial de la Salud, 2006), por lo que podría inferirse que para disminuirlo se prescribe incapacidad no solo para restaurar el estado de salud, sino como medida preventiva para evitar más casos en las oficinas. En cuanto a las incapacidades por lesiones osteomusculares, se recomienda reposo para la recuperación de la parte afectada y lograr en conjunto con el tratamiento terapéutico una incorporación laboral pronta (Melguizo, 1998; Royo-Bordonada, 1999; Castroviejo, 2008; Caja Costarricense de Seguro Social, 2011). Las extendidas por enfermedades digestivas están ligadas a la mala dieta y deficiente técnica del lavado de manos (Organización Mundial de la Salud, 2006).

Consulta por departamento: La policonsulta registrada en algunos departamentos u oficinas, podría deberse a estrés laboral, hacinamiento y sobrecarga de funciones; estas condiciones se mantienen en casi todas las dependencias. Interesante que algunos departamentos consultan menos y equilibran el promedio de consultas anuales por usuario (Arce \& Monge, 2008). Para poder determinar las causas que provocan un aumento de consultas en unos departamentos más que otros, debe de realizarse otro estudio, el cual contemple los riesgos ocupacionales mencionados. 


\section{CONCLUSIONES}

La mitad de los usuarios asistieron en promedio seis veces al consultorio médico.

La población se encuentra dividida en dos grandes grupos de edad los adultos jóvenes de 18 a 37 años y los de 38 años a más de 58 años.

La cantidad de consultas se mantiene estable a lo largo del año, presentando descensos por vacaciones institucionales y ascensos por las campañas preventivas de ultrasonidos y cáncer de seno, por lo cual se cumple la hipótesis de los ciclos mensuales y semanales.

Coinciden con lo reportado por la Caja Costarricense del Seguro Social las enfermedades de las vías respiratorias, las consultas sin hallazgo patológico, las enfermedades osteomusculares, las enfermedades endocrinas y metabólicas; no coinciden las enfermedades digestivas reportadas en este servicio en contraste con las del sistema circulatorio reportado por el sistema de seguridad social nacional. Por lo que podría afirmarse que se cumple la segunda hipótesis en que las variables de ocupación laboral y nivel socioeconómico no generan diferencias significativas del personal académico en relación a la población nacional.

La segunda causa de consulta reportada que corresponde a exámenes médicos de rutina, demuestra que existe conciencia por parte del usuario de la responsabilidad para con su salud.

Las mujeres consultan en mayor proporción que los hombres, lo cual demuestra que las mujeres supervisan más su estado de salud.

Se mantiene la hipótesis de que la ocupación laboral y el nivel socioeconómico no interfieren en el patrón de morbilidad de la universidad con respecto al patrón nacional.

Se mantiene la hipótesis de que la consulta externa y su morbilidad en esta universidad estatal de Costa Rica presenta ciclos semanales $y$ anuales.

\section{RECOMENDACIONES}

Realizar un estudio de salud ocupacional que incluya los riesgos mencionados en esta investigación: hacinamiento, recarga de funciones, riesgos ambientales $y$ ergonómicos.

Implementar un programa de medicina preventiva para disminuir las enfermedades de la vías respiratorias que abarque: la colocación de la vacuna de la influenza a grupos de riesgo, revisión de filtros de aires acondicionados, educación acerca del protocolo de estornudo y tos, insistencia en el lavado de manos así como en las medidas de limpieza de implementos de oficina de uso compartido y cambios en estilos de vida saludables.

Implementación de un programa preventivo de cuidado osteomuscular que abarque higiene postural.

La morbilidad derivada de enfermedades endocrinas y metabólicas o nutricionales, así como, las del aparato digestivo, podrían ser disminuidas con apoyo nutricional y con acompañamiento del galeno, dando una intervención integral en este tipo de enfermedades.

\section{AGRADECIMIENTOS}

A Víctor Méndez Estrada y a Ligia Bermúdez Mesén por su apoyo en esta investigación.

\section{REFERENCIAS}

Alfieri, R., \& Challem, J. (2007). Guías prácticas de Salud: antienvejecimiento. España, Madrid: Ediciones Nowtilus.

Arce, L., \& Monge, J. (2008). Morbilidad y ciclos temporales de la consulta externa de la Universidad Estatal a Distancia de Costa Rica. Revista Ciencias del Movimiento Humano, 5(1), 1-11.

Arce, L., \& Monge, J. (2011). Reporte sobre lavado de manos y enfermedades relacionadas en usuarios de los baños públicos de una universidad costarricense. Enfermería en Costa Rica, 32(1), 19-23.

Caja Costarricense de Seguro Social. (2002a). Cuarta encuesta nacional sobre las características de los usuarios de los servicios de la consulta externa. San José, Costa Rica: CCSS.

Caja Costarricense de Seguro Social. (2002b). Moderinización : comunicación para un mejor cambio. San José, Costa Rica: Boletín Caja Costarricense de Seguro Social.

Caja Costarricense de Seguro Social. (2011). Modificación del Reglamento de otorgamiento de incapacidades y licencias a los beneficiarios del seguro social. San José, Costa Rica: CCSS.

Castroviejo, S. B. R. (2008). Valoración de la incapacidad laboral. Rehabilitación, 42(6), 291-314.

Esquivel, V., \& Alvarado, M. (2009). Estado nutricional de mujeres con sobrepeso y obesidad del área de cobertura de atención integral de salud (PAIS), 2006. Acta Médica Costarricense, 51(4), 222-228.

Lorca, J. (2013). Construyendo la innovación social, desde abajo. Bloomington, Indiana: Palibrio. Recuperado de http:// goo.gl/wG0L56

Linero, E. M., \& Rodríguez, R. (2012). Prevalencia de síntomas osteomusculares en el personal de salud de dos 
instituciones prestadores de salud en la ciudad de Bogotá, durante el año 2012. Recuperado de www.ergonomia.cl

Melguizo, M. (1998). La contra reforma de la incapacidad laboral. Atención Primaria, 22, 69-90.

Ministerio de Salud. (2010). Memoria Institucional 2010. San José, Costa Rica: Ministerio de Salud.

Moya, L. (2003). Cambios en la morbilidad y mortalidad por edad y sexo, Costa Rica, 1987, 1992,1997 y 2002. Sección de Información Biomédica de la Caja Costarricense de Seguro Social, San José, Costa Rica.

Oliva, J. (2009). Obesidad y calidad de vida relacionada con la salud. Working papers, Universidad de Castilla- La Mancha, 1-26.

Organización Mundial de la Salud. (2006). Ambientes saludables y prevención de enfermedades. Francia: OMS:
Organización Mundial de la Salud. (2009). Código Internacional de Enfermedades . España: OMS

Organización Mundial de la Salud. (2011). Los jóvenes y riesgos sanitarios. Ginebra: OMS

Ortiz-Barboza, A. (2010). La Salud Pública en Costa Rica, retos y perspectivas. San José, Costa Rica: Universidad de Costa Rica.

Royo-Bordonada, M. Á. (21 de Mayo de 1999). La duración de la incapacidad y sus factores asociados. Gaceta Sanitaria, 13, 177-184.

Sepúlveda, M. I. (1994). La Salud del Adolescente y Joven. Boletin Escuela de Medicina Pública.Universidad Católica de Chile, 23, 15-17.

Vaca, W. L. (2003). Estadística descriptiva con énfasis en salud pública. Bolivia: Grupo Editorial, La Hoguera. 
APÉNDICE 1

Distribución por edad según dependencia, 2009-2012 (porcentaje)

\begin{tabular}{lccccc}
\multicolumn{1}{c}{ Dependencia } & De 18 a 27 & De 28 a 37 & De 38 a 47 & De 48 a 57 & 58 y más \\
Vicerrectoría Académica & 14,3 & 28,0 & 24 & 25 & 7,5 \\
Vicerrectoría de Investigación & 26,1 & 35,9 & 16,2 & 14,9 & 6,8 \\
Vicerrectoría Ejecutiva & 14,5 & 32,1 & 27,5 & 23,2 & 2,6 \\
Vicerrectoría de Planificación & 10,4 & 46,5 & 22,4 & 17,8 & 2,9 \\
Rectoría & 20,5 & 40,1 & 20,5 & 18,8 &, 1 \\
Aseuned & 27,1 & 42,6 & 24,8 & 5,4 &, 0 \\
Otras dependencias & 10,5 & 29,6 & 22,4 & 35,4 & 2,1 \\
Pensionado &, 0 &, 0 &, 0 & 26,8 & $\mathbf{7 3 , 2}$ \\
No disponible & 24,2 & 34,4 & 19,3 & 16,8 & 5,3 \\
Total & $\mathbf{1 4 , 9}$ & $\mathbf{3 0 , 7}$ & $\mathbf{2 4 , 9}$ & $\mathbf{2 4 , 0}$ & $\mathbf{5 , 5}$ \\
\hline
\end{tabular}

APÉNDICE 2

Correlación por edad y diagnóstico según CIE10( porcentaje)

\section{Grupo CIE}

Ciertas enfermedades infecciosas y parasitarias Neoplasias

Enfermedades de la sangre y de los órganos hematopoyéticos y otros trastornos que afectan el mecanismo de la inmunidad

Enfermedades endocrinas, nutricionales y metabólicas

Trastornos mentales y del comportamiento

Enfermedades del sistema nervioso

Enfermedades del ojo y sus anexos

Enfermedades del oído y de la apófisis mastoides

Enfermedades del sistema circulatorio

Enfermedades del sistema respiratorio

Enfermedades del aparato digestivo

Enfermedades de la piel y el tejido subcutáneo

Enfermedades del sistema osteomuscular y del tejido conectivo

Enfermedades del aparato genitourinario

Embarazo, parto y puerperio

Malformaciones congénitas, deformidades y anomalías cromosómicas

Síntomas, signos y hallazgos anormales clínicos y de laboratorio, no clasificados en otra parte

Traumatismos, envenenamientos y algunas otras consecuencias de causa externa

Causas extremas de morbilidad y de mortalidad

Factores que influyen en el estado de salud y contacto con los servicios de salud

\section{Grupo de edad}

De 18 a $27 \quad$ De 28 a $37 \quad$ De 38 a 47 De 48 a $57 \quad 58$ y más

$\begin{array}{lllll}16,8 & 25,5 & 24,2 & 27,8 & 5,7\end{array}$

$\begin{array}{lllll}18,9 & 29,4 & 19,4 & 26,4 & 6,0\end{array}$

$\begin{array}{lllll}8,9 & 34,7 & 30,5 & 22,5 & 3,3\end{array}$

$\begin{array}{lllll}14,4 & 28,3 & 25,9 & 25,8 & 5,6\end{array}$

$\begin{array}{lllll}17,6 & 29,4 & 23,5 & 23,6 & 5,8\end{array}$

$\begin{array}{lllll}17,3 & 30,4 & 18,5 & 27,8 & 6,0\end{array}$

$\begin{array}{lllll}18,5 & 27,5 & 26,3 & 23,5 & 4,1\end{array}$

$\begin{array}{lllll}16,1 & 26,5 & 24,0 & 26,3 & 7,1\end{array}$

$\begin{array}{lllll}16,0 & 32,2 & 22,7 & 23,9 & 5,2\end{array}$

$\begin{array}{lllll}14,9 & 32,5 & 26,4 & 21,4 & 4,8\end{array}$

$\begin{array}{lllll}15,7 & 35,8 & 24,5 & 19,6 & 4,4\end{array}$

$\begin{array}{lllll}15,4 & 32,5 & 25,6 & 21,0 & 5,5\end{array}$

$\begin{array}{lllll}13,0 & 32,0 & 28,3 & 22,3 & 4,4\end{array}$

$\begin{array}{lllll}15,4 & 28,5 & 25,9 & 24,2 & 6,0\end{array}$

$\begin{array}{lllll}12,2 & 31,7 & 12,2 & 31,7 & 12,2\end{array}$

$\begin{array}{lllll}8,6 & 22,9 & 25,7 & 31,4 & 11,4\end{array}$

$\begin{array}{lllll}13,7 & 34,8 & 26,2 & 20,3 & 4,9\end{array}$

$\begin{array}{lllll}14,5 & 33,8 & 24,4 & 22,0 & 5,3\end{array}$

$\begin{array}{lllll}15,8 & 33,2 & 18,9 & 24,2 & 7,9\end{array}$

$\begin{array}{lllll}14,4 & 25,8 & 21,4 & 31,1 & 7,4\end{array}$


APÉNDICE 3

Correlación entre el sexo y la morbilidad según CIE10 (porcentaje)

\begin{tabular}{|c|c|c|}
\hline \multirow{2}{*}{ Grupo CIE } & \multicolumn{2}{|c|}{ Sexo } \\
\hline & Mujer & Hombre \\
\hline Ciertas enfermedades infecciosas y parasitarias & 61,6 & 38,4 \\
\hline Neoplasias & 66,7 & 33,3 \\
\hline $\begin{array}{l}\text { Enfermedades de la sangre y de los órganos hematopoyéticos y otros trastornos que afectan el mecanismo } \\
\text { de la inmunidad }\end{array}$ & 61,5 & 38,5 \\
\hline Enfermedades endocrinas, nutricionales y metabólicas & 61,5 & 38,5 \\
\hline Trastornos mentales y del comportamiento & 63,3 & 36,7 \\
\hline Enfermedades del sistema nervioso & 63,0 & 37,0 \\
\hline Enfermedades del ojo y sus anexos & 62,6 & 37,4 \\
\hline Enfermedades del oído y de la apófisis mastoides & 60,1 & 39,9 \\
\hline Enfermedades del sistema circulatorio & 61,1 & 38,9 \\
\hline Enfermedades del sistema respiratorio & 62,2 & 37,8 \\
\hline Enfermedades del aparato digestivo & 61,8 & 38,2 \\
\hline Enfermedades de la piel y el tejido subcutáneo & 63,5 & 36,5 \\
\hline Enfermedades del sistema osteomuscular y del tejido conectivo & 64,4 & 35,6 \\
\hline Enfermedades del aparato genitourinario & 62,1 & 37,9 \\
\hline Embarazo, parto y puerperio & 46,3 & 53,7 \\
\hline Malformaciones congénitas, deformidades y anomalías cromosómicas & 71,4 & 28,6 \\
\hline Síntomas, signos y hallazgos anormales clínicos y de laboratorio, no clasificados en otra parte & 63,1 & 36,9 \\
\hline Traumatismos, envenenamientos y algunas otras consecuencias de causa externa & 60,4 & 39,6 \\
\hline Causas extremas de morbilidad y de mortalidad & 58,9 & 41,1 \\
\hline Factores que influyen en el estado de salud y contacto con los servicios de salud & 59,9 & 40,1 \\
\hline Total & $61,9 \%$ & $38,1 \%$ \\
\hline
\end{tabular}

APÉNDICE 4

\begin{tabular}{lcc} 
& Grupo & Porcentaje \\
Grupo 1 & 18,0 \\
Grupo 2 & 21,6 \\
Grupo 3 & 25,1 \\
Grupo 4 & 35,3 \\
Total & 100 \\
\hline
\end{tabular}

